France, 1862, Pl. 13) gebrachten wegen der beigefügten Abbildungen die besten sind. Wir geben daher nur die Abbildung des Kopfes.

Die allenfalls mit der neuen Art zu vergleichenden südamerikanischen Arten lassen sich wie folgt unterscheiden:

1 (2) Deckenspatien gewölbt, Deckenstreifen mäßig tief, undeutlich punktiert, Schildchen doppelt so lang wie breit, streifenförmig, alle Beine, mit Ausnahme der Tarsen, gelb. Brasilien.

ampullula Gerst. ${ }^{1}$ )

2 (1) Deckenspatien abgeflacht, Schildchen punktförmig.

3 (4) Deckenstreifen grob, fast so breit wie die Spatien, Halsschild scheinbar glatt, mit zerstreuten börstchentragenden Punkten, vor dem Schildchen mit scharfem Längseindruck, alle Beine ganz rotgelb. Peru . . . . . . . . . pyriforme Kirsch

4 (3) Deckenstreifen viel schmäler wie die Spatien.

5 (6) Der 1. und 2. Streifen jederseits neben der Naht viel kräftiger als die übrigen, Halsschild äußerst fein, undeutlich punktiert, Vorderschienen und Mittelschienen rotgelb. Columbien

rhomboideum Gerst. ${ }^{1}$ )

6 (5) Alle Deckenstreifen untereinander gleich kräftig, Halsschild spärlich, aber kräftig punktiert, Fühlerschaft und Vorderschienen, zuweilen auch das 1. Geißelglied und die Schenkel, hell bräunlichgelb, Schildchen verrundet viereckig. Rio Janeiro . . zikani sp. n.

\title{
Labidostoma brasiliense Sellnick n. sp. (Acar.)
}

Von Max Sellnick (Lötzen, Ostpr.)

Mit 1 Textfigur.

Canestrini und Fanzago veröffentlichen 1877 (Atti Ist. Veneto, s. 5, v. 4, p. 52) die Beschreibung einer Milbenart Nicoletia cornuta. Kramer beschreibt 1879 (Arch. Naturg., Jahrg. 45, v. 1, p. 13) eine Milbe, welche zu derselben Gattung gehört, unter dem Namen Labidostomma ${ }^{2}$ ) luteum. - Der Name Nicoletia ist bereits 1840 von Gervais für eine Gattung der Lepismatidae vergeben. Nach den Regeln der Nomenklatur hätte nun Labidostoma an seine Stelle treten müssen. Canestrini nennt aber 1882 (Atti Soc. Veneto-Trent., v. 8, p. 136) die Gattung Nicoletiella. Dieser Name muß also fallen. - O udemans

$\left.{ }^{1}\right)$ Ent. Zeit. Stettin XV, 1854, 246 u. 255.

$\left.{ }^{2}\right)$ corr. Labidostoma. 
behauptet (Ent. Ber., v. 1, p. 83, 1903 und v. 2, p. 38, 1906), daß Nicoletiella cornuta Can. u. Fanz. identisch mit Acarus denticulatus Schrank (Beyträge zur Naturg., Augsburg 1776) sei. Es wäre möglich. Aber ebensogut könnte Schranks Tier auch L. luteum Kramer sein. Ich bin für Beibehalten des Artnamens cornuta Can. u. Fanz. - Zur Familie der Labidostomidae gehören 2 Gattungen, Labidostoma Kramer 1879 und Eunicolina Berlese 1911. Die Gattung Ceratoacarus Ewing 1913 ist wohl ein Synonym von Labidostoma.

Die zu den beiden Gattungen gehörigen Arten sind, soweit mir bekannt, die folgenden:

Labidostoma Kramer. 1879, Arch. Naturg., Jahrg. 45, v. 1, p. 13.

1. L. cornutum (Can. u. Fanz.), 1877 (Nicoletia cornuta), Lit. s. oben. Italien. Länge $1 \mathrm{~mm}$. Typus der Gattung.

2. L. luteum Kramer, 1879 (Labidostomma l.), Lit.s. oben. Deutschland. Länge $0,7 \mathrm{~mm}$.

3. L. neotropicum (Stoll). 1893 (Nicoletiella neotropica), Biol. Centr.Amer., Arach. p. 27. Guatemala. Länge 0,5 mm.

4. L. corcyraeum Berlese. 1911, Redia, v. 7, p. 429. Insel Corcyra. Länge $0,75 \mathrm{~mm}$.

5. L. integrum Berlese. 1911, Redia, v. 7, p.429. Umbria, Italien. Länge $0,62 \mathrm{~mm}$.

6. L. pacificum (Ewing). 1913 (Ceratoacarus pacificus), J. Ent. Zool., v. 5, p. 128. Oregon, Nord-Amerika. Länge 1,56 mm.

Dazu kommt als neue Art:

7. L. brasiliense Sellnick. Brasilien. Länge $0,84 \mathrm{~mm}$.

Eunicolina Berlese. 1911, Redia, v. 7, p. 430.

1. E. tuberculata Berlese. 1911, Redia, v. 7, p. 430. Italien. Länge $0,7 \mathrm{~mm}$. Typus der Gattung.

Labidostoma brasiliense Sellnick nov. spec.

Farbe gelb, nach hinten zu ins Olivgrüne übergehend. Tibia und Tarsus I hellgelb, die anderen Glieder dieses Beines auffallend dunkelolivgrün. Die anderen Beine schwach olivgrün. Oberfläche des Körpers vielfach gefurcht. Bemerkenswert sind 3 flache Längseindrücke, welche etwas vor der Mitte des Rückens beginnen und bis zu Dreiviertel der Körperlänge nach hinten reichen. Hinter ihnen liegen 2 kleine rundliche Eindrücke. Diese und die 3 Längsgruben sind fein gepunktet. Der übrige Teil des Rückens ist mit rundlichen Gruben dicht bedeckt. Über Bein III liegt an der Seite des Körpers ein heller Fleck, der wohl allen Arten der Gattung eigentümlich ist. Sein Zweck ist nicht untersucht. Ewing bezeich net ihn als Auge, wie auch einen medianen Fleck 
am Vorderrande des Rückens. Ein solcher ist bei brasiliense ebenfalls angedeutet. Ein vorspringender Chitinzapfen, wie ihn $L$. luteum neben dem Seitenfleck aufweist, ist bei brasiliense nicht vorhanden. Die Behaarung des Rückens ähnelt der von L. luteum. Drei Haarpaare auf seinem vorderen Teile sind vorwärts gerichtet: ein kürzeres einfaches an der Außenseite des Schulterhornes, ein fast doppelt so langes, in der

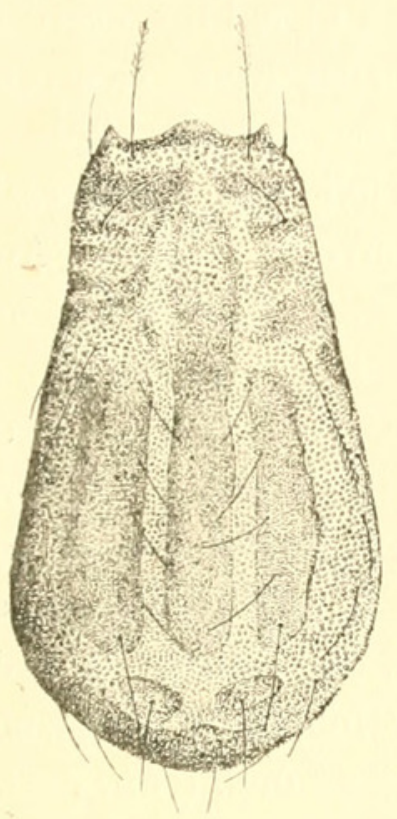
vorderen Hälfte beborsteltes, auf der Innenseite des Hornes und weiter zurückstehend ein einfaches kurzes, welches ein wenig nach innen geneigt ist. Hinter Bein II beginnt eine Randreihe und zwei Mittelreihen einfacher Borsten. Zwischen Rand und Mittelreihe steht über Bein II ein langes beborsteltes Haar. Die beiden beborstelten Haarpaare entspringen aus Becherchen, welche den Pseudostigmen der Oribatidae sehr ähnlich sehen. Während die Vorderkante des Hypostoms von L. luteum eine fast gerade Linie bildet, zeigt sie bei $L$. brasiliense deutlich vorspringende Seitenecken. Mandibeln mit einem Höcker, von welchem ein langes Haar entspringt. Länge des Körpers ohne Mandibeln $840 \mu$., Breite an den Schultern $275 \mu$., hinter Bein IV $460 \mu$.

Eine Anzahl Exemplare von Herrn J. F. Zikán bei Passa Quatro, Sul de Minas, Brasilien, gesammelt.

\section{Einige species novae der Anthribicen-Gattung Zygaenodes Pascoe. (Col.)}

Von Dr. Paul Wolfrum, Wefensleben (Bezirk Magdeburg).

Das bearbeitete Material stammt in der Hauptsache von C. Fuller Baker (Hofrat Dr. K. M. Heller); einige Stücke vom Berliner zoologischen Museum sind mit einbezogen. Von dem bekannten Zygaenodes Wollastoni Pasc. lagen mir 4 ổ und 4 \&̊ vor. Die Originalbeschreibung Pascoes (The Annals and Magazine of Nat. Hist. (III), vol. IV, 1859, p. 328) ist auf fo+ gegründet und nicht, wie Lacordaire (Genera des Coléoptères, VII, p. 542-544) annimmt, auf oto $^{\circ}$. Ich stelle daher die sekundären Geschlechtsmerkmale des Wollastoni Pasc. folgendermaßen gegenüber. ô. Fühler kürzer, die Mitte der Flügeldecken erreichend, Keule kräftiger. Die Augenstiele, von vorne gesehen, gegen das Auge gerade abgeschnitten, etwa $1 / 4$ so lang wie der Längsdurchmesser der Augen. Die Augen hoch gewölbt, halbkugelförmig. - ․ Fühler sehr 


\section{$2 \mathrm{BHL}$ Biodiversity Heritage Library}

1922. "Labidostoma brasiliense Sellnick n. sp. (Acar.)." Entomologische Mitteilungen 11, 54-56. https://doi.org/10.5962/bhl.part.3334.

View This Item Online: https://www.biodiversitylibrary.org/item/39843

DOI: https://doi.org/10.5962/bhl.part.3334

Permalink: https://www.biodiversitylibrary.org/partpdf/3334

\section{Holding Institution}

Smithsonian Libraries

\section{Sponsored by}

Smithsonian

\section{Copyright \& Reuse}

Copyright Status: NOT_IN_COPYRIGHT

This document was created from content at the Biodiversity Heritage Library, the world's largest open access digital library for biodiversity literature and archives. Visit BHL at https://www.biodiversitylibrary.org. 